\title{
Jugendkriminalität in sozialen Kontexten - Zur Rolle von Wohngebieten und Schulen bei der Verstärkung von abweichendem Verhalten Jugendlicher
}

\section{Dietrich Oberwittler}

M ax-Planck-Institut für ausländisches und internationales Strafrecht, Abt. Kriminologie, Freiburg

erscheint in:

Dollinger, Bernd; Schmidt-Semisch, Henning (Hg.) (2018), Handbuch J ugendkriminalität. Interdisziplinäre Perspektiven. 3. Auflage, Wiesbaden: Springer VS, S. 297-316.

Print ISBN 978-3-531-19952-8; Online ISBN 978-3-531-19953-5

DOI 10.1007/978-3-531-19953-5_15 


\section{Dietrich Oberwittler}

\section{Jugendkriminalität in sozialen Kontexten - Zur Rolle von Wohnge- bieten und Schulen bei der Verstärkung von abweichendem Verhal- ten Jugendlicher}

Dass Einflüsse der Lebensumwelten einen positiven oder negativen Beitrag zur Entwicklung von Kindern und Jugendlichen leisten und auch an der Entstehung von Delinquenz beteiligt sein können, ist keine überraschende Erkenntnis, auch wenn sie in den Forschungsansätzen zu Jugenddelinquenz lange Zeit keine angemessene Aufmerksamkeit gefunden hat. Effekte sozialräumlicher Kontexte auf Individuen zu untersuchen ist aufwändig und fügt der ohnehin schwierigen Theoriebildung und empirischen Analyse eine weitere Komplexitätsstufe hinzu. Nach rund zwei Jahrzehnten intensiver Forschung ist jedoch ein teilweise recht gefestigtes Bild von der Rolle sozialräumlicher Kontexte entstanden, das man ganz knapp so zusammenfassen kann: Konzentrierte soziale Benachteiligungen in Wohngebieten und Schulen können Delinquenz verstärken, wobei diese Effekte meist kleiner sind als die individueller und familiärer Risikofaktoren. Kontexteffekte sind immer das Ergebnis von Wechselwirkungen der sozialen Umwelt mit handlungsmächtigen und mit unterschiedlichen Ressourcen und Präferenzen ausgestatteten Jugendlichen und ihrer Familien, sie gelten daher nicht für alle gleichermaßen, z.B. für Mädchen mehr als für Jungen. Und ein starker Wohlfahrtsstaat kann die negativen Effekte sozialräumlicher Benachteiligungen abfedern. Dieser Beitrag bietet einen knappen Überblick über die theoretischen Grundlagen und empirischen Ergebnisse dieser Forschung, verzichtet aber auf viele Differenzierungen und methodische Aspekte (vgl. Oberwittler 2013 für eine ausführliche Darstellung). ${ }^{1}$

Die Beobachtung, dass Kriminalität und Gewalt in den Armutsvierteln der Großstädte grassieren, hat eine sehr lange Tradition, die bis in das 19. Jahr-

\footnotetext{
${ }^{1}$ Um den Umfang des Kapitels konstant zu halten wurde hier auf die Zitierung vieler älterer Veröffentlichungen zugunsten der aktuellen Literatur verzichtet. Diese älteren Veröffentlichungen finden sich in meinem Beitrag zur ersten und zweiten Auflage dieses Handbuches.
} 
hundert zurück reicht. Die stadtsoziologische ,Chicago School' entwickelte in der Mitte des 20. Jahrhunderts die Theorie der sozialen Desorganisation und legte damit eine Basis für die weitere Forschung über großstädtische Viertel mit hoher Kriminalitätsbelastung. Clifford Shaw und Henry McKay (1969 [1942]) stellten fest, dass bestimmte, durch Armut, ethnische Heterogenität und hohe Fluktuation geprägte Stadtviertel Chicagos über lange Zeiträume hinweg sehr hohe Jugendkriminalitätsraten aufwiesen (,delinquency areas'), und erklärten dies mit der mangelnden Fähigkeit der Bewohner, eine effektive informelle Sozialkontrolle über die Jugendlichen auszuüben, sowie mit der fortwährenden Weitergabe subkultureller Orientierungen von einer Jugendgeneration an die nächste. Ihre Erklärung der Jugendkriminalität zielte also explizit auf die kollektiven Eigenschaften der Stadtviertel, nicht auf die individuellen Eigenschaften der jugendlichen Bewohner oder ihrer Familien. In Deutschland griffen Karl-Dieter Opp (1968) und Detlev Frehsee (1979) diesen Ansatz auf und stellten die Bedeutung der Subkultur in den Mittelpunkt, erkannten jedoch auch die methodischen Beschränkungen des klassischen Desorganisationsansatzes. Erst mit der großflächigen Verbreitung von Jugendbefragungen zur selbstberichteten Delinquenz, Bewohnerbefragungen zu sozialen Prozessen im Wohnquartier sowie mit der Entwicklung der statistischen Mehrebenenanalyse zur Trennung von Individual- und Kontexteffekten hat dieses Forschungsthema seit den 1990er Jahren wieder einen Aufschwung erlebt. Diese jüngere Forschung spielt sich allerdings ganz überwiegend außerhalb Deutschlands, vor allem in den USA, Skandinavien, Großbritannien und den Niederlanden ab (Bottoms 2012; Nieuwenhuis/Hooimeijer 2016, Oberwittler/Rabold/Baier 2013; Sharkey/Faber 2013; Sampson/Morenoff/Gannon-Rowley 2002).

Dass sich sozialräumliche Lebensumwelten von Jugendlichen unterscheiden, ist in erster Linie der grundlegenden Tatsache der sozialen (und ethnischen) Segregation geschuldet, die über verschiedene Mechanismen des Wohnungsmarktes, der Organisation des Bildungswesens etc. dazu führt, dass Familien in ähnlichen sozialen Lebenslagen auch räumlich eher in Nachbarschaft zueinander wohnen, und dass Jugendliche ähnlicher sozialer und ethnischer Herkunft eher die gleichen Schulen besuchen (Farwick 2012; Häußermann 2008; Oberwitt- 
ler 2007a; van Ham/Manley 2012). Die schärfste Form der Segregation stellen die so genannten sozialen Brennpunkte in den Großstädten dar, also Stadtviertel mit hohen Konzentrationen sozial benachteiligter Bewohner. Der wirtschaftliche Strukturwandel mit einem Verlust klassischer Industriearbeit und auch die Einwanderung niedrigqualifizierter Migration verstärken diese Tendenzen der sozialen Exklusion, also der mangelnden gesellschaftlichen Integration in verschiedenen Bereichen wie Bildung, Kultur und Politik (Murie/Musterd 2004). Kinder und Jugendliche, deren Sozialisation noch nicht abgeschlossen ist, sind davon vermutlich in besonderem Maße betroffen (Duncan/Murnane 2011; Klocke/ Hurrelmann 1998).

\section{Theoretische Erklärungsansätze}

Die Wirkung von Sozialräumen auf Menschen wird in den theoretischen Erklärungsansätzen in erster Linie als eine Frage der sozialen Organisation, nicht der physischen oder baulichen Struktur, verstanden (Bursik 1988; Wikström 2007). Sozialräumliche Theorien abweichenden Verhaltens verfolgen keine gänzlich neuen Fährten, sondern lehnen sich sehr eng an bestehende kriminalsoziologische Theorien, vor allem Kontroll- und Lerntheorien, an, und berücksichtigen in jüngster Zeit verstärkt auch Elemente von Handlungs- und Entscheidungstheorien, um der Bedeutung von Handlungsmacht (agency) gerecht zu werden. Sozialräumliche Theorien nehmen die kollektiven Eigenschaften von sozialen Gruppen in den Blick, von denen angenommen wird, dass sie mehr sind als die Summe der Eigenschaften der Individuen und daher eigenständige Wirkungen entfalten. Verschiedene soziologische Theoretiker wie Coleman (1990) und Boudon (1998) haben Wirkungspfade von kollektiven Eigenschaften auf individuelles Verhalten im Rahmen von Mikro-Makro-Mikro-Modellen postuliert (vgl. auch Esser 2002; Hess/Scheerer 2004).

Der populärste Ansatz baut auf lern- und subkulturtheoretischen Erkenntnissen auf, nach denen delinquentes Verhalten durch den Kontakt mit anderen delinquenten Jugendlichen 
erlernt und verstärkt wird (Akers/Jensen 2003). Die Rolle der Gleichaltrigen - vor allem der delinquenten peers - in der Entwicklung von Jugenddelinquenz ist in den letzten Jahren nach einer langen Phase der Unterbewertung wieder neu ,entdeckt' und vor allem mit netzwerkanalytischen Mitteln intensiver erforscht worden. Deutsche und internationale Studien haben gezeigt, dass delinquente Freunde einen verstärkenden Effekt auf Jugenddelinquenz haben (Beier 2014; Dishion/Tipsord 2011, Gerstner/Oberwittler 2015; Haynie/Kreager 2013; Rabold/Baier 2010; Zimmerman/Messner 2011). Dies gilt auch, wenn man in Längsschnittstudien den starken Effekt der Selbstselektion in delinquente Freundesnetzwerke berücksichtigt (Barfeldt et al. 2008; Haynie/Osgood 2005; Weerman 2011; Steglich et al 2010; gegenteilig Knecht et al. 2010). Die sozialräumliche Dimension fügt diesem Mechanismus insofern Brisanz hinzu, als mit der Konzentration sozial benachteiligter Jugendlicher in Schulen und Wohngebieten Kontakte mit anderen Jugendlichen wahrscheinlicher werden, die gleiche individuelle Risikofaktoren und delinquente Neigungen aufweisen. Für das Phänomen delinquenter Banden und „Gangs“ ist die geographische Nähe geradezu eine Bedingung (Papachristos et al. 2013; Schaefer 2012). Crane (1991: 1226) hat diesen Beeinflussungseffekt in Anlehnung an die Übertragung ansteckender Krankheiten als ,the epidemic theory of ghettos“ bezeichnet. Nach dieser Vorstellung entwickelt sich in benachteiligten Wohnquartieren und in Schulen eine delinquente Subkultur, indem abweichende Normen, wie z.B. die Anwendung von Gewalt in Konfliktsituationen, seltener abgelehnt und häufiger akzeptiert oder sogar erwartet werden. Anderson (1999) spricht von einem auf Gewalt und Ehre basierenden „,code of the street“, der die Verhaltenserwartungen in den Armenghettos der U.S.-amerikanischen Großstädte prägt (vgl.

bereits Frehsee 1979). Inzwischen wurde diese recht weitgehende Annahme der Dominanz delinquenter Normen zugunsten der Überlegung aufgegeben, dass in den sozial benachteiligten Wohnquartieren die Normheterogenität wächst (Berg et al. 2012; Friedrichs 1997; Harding 2010; Small/Harding/Lamont 2010). Dies erfordert von den Jugendlichen einerseits 
eine Auswahl zwischen Handlungsalternativen, andererseits erschwert die Normheterogenität die Verständigung über und Realisierung von gemeinsamen Zielen, z.B. der entschiedenen Kontrolle jugendlichen Fehlverhaltens im öffentlichen Raum durch die Bewohner (siehe unten).

Weitere negative Effekte der räumlichen Konzentration von sozialen Benachteiligungen, insbesondere von Arbeitslosigkeit und Armut, liegen nach Wilson (1997, vgl. Friedrichs/Galster/Musterd 2003; Galster 2012) in dem Fehlen von positiven Rollenvorbildern z.B. der erfolgreichen Bildungs- und Berufskarriere. Wenn angesichts schlechter Bildungsund Arbeitsmarktchancen die Aussichten auf eine positive Berufskarriere gering sind, dürften die Bindungen an die konventionelle Gesellschaft und die Legitimität ihrer Normen leiden und die Attraktivität illegaler Erwerbsmöglichkeiten steigen (Grogger 1998). Beides ist ein geeigneter Nährboden nicht nur für Kriminalität, sondern auch für kollektive Gewalt, wie sie etwa in den französischen Vorstädten zu beobachten ist.

Ein zusätzlicher, erstmals in der klassischen Studie von Shaw und McKay ausformulierter Ansatz hebt auf die mangelnde informelle Sozialkontrolle der Jugendlichen durch die erwachsenen Bewohner ab. Eine hohe Fluktuation, ethnische Heterogenität sowie Armutskonzentration in den benachteiligten Wohnquartieren verhindern demnach über die fehlende soziale Kohäsion der Bewohner eine effektive Kontrolle und führen zu sozialer Desorganisation. Dieser Ansatz wurde in den 1990er Jahren unter dem Begriff der kollektiven Wirksamkeit (,collective efficacy') weiterentwickelt und hat eine große Verbreitung gefunden (Sampson/Raudenbush/Earls 1997; vgl. Friedrichs/Oberwittler 2007; Wickes et al. 2016). So wurde er auch auf die Organisation von Schulen übertragen (Payne et al. 2003; Wilbers 2004): In effektiv organisierten Schulen sorgen Lehrer durch ein positives Schulklima und gemeinschaftliche Strategien dafür, dass Gewalt und anderes Fehlverhalten kontrolliert werden und sich nicht in dem Maße ausbreiten, wie es andernfalls zu erwarten wäre. Einen ähnlichen, sowohl Wohnquartiere als auch Schulen umfassenden Aspekt hat Coleman (1988) mit dem Begriff der ,intergenerational closure' herausgestrichen: Der Kontakt von Eltern mit anderen Eltern über ihre Kinder eröffnet durch Austausch und gegenseitige Unterstützung in schulischen und anderen Belangen zusätzliche, extra-familiäre Ressourcen für eine gelingende Sozialisation (Valdimarsdottir/Bernburg 2015). 
Ging es in der neueren Forschung anfangs darum, die Existenz der theoretisch vorausgesagten Auswirkungen sozialräumlicher Kontexte auf Jugendliche empirisch zu belegen oder zu widerlegen, so wurde mit ihrem Fortgang schnell deutlich, dass die pauschale Annahme solcher Effekte auf ,die' Jugendlichen in benachteiligten Sozialräumen eine Vereinfachung darstellt, die den komplexen Realitäten nicht angemessen ist (Oberwittler 2013; Sharkey/Faber 2014). Denn Jugendliche sind

keine passiven Opfer ihrer Lebensumwelt, sondern ,produktiv Realität verarbeitende Subjekte' (Hurrelmann 2006), die sich aktiv mit ihrer Lebensumwelt auseinandersetzen und unterschiedlich auf sozialräumliche Bedingungen reagieren. Dies beginnt mit der wichtigen Frage nach der räumlichen Ausrichtung der Aktionsräume und Freundesnetzwerke von Jugendlichen, die als Ausdruck individueller Präferenzen verstanden werden kann (Arum 2000; Miltenburg 2015; Oberwittler 2004b; Wikström und Sampson 2003, siehe unten). Das eigene Wohngebiet stellt zwar eine Gelegenheitsstruktur für Kontakte dar, ist aber keine Insel, auf die Jugendliche notwendigerweise beschränkt sind.

Die von Per-Olof Wikström entwickelte „Situational Action Theory“ (SAT) nimmt diese Überlegungen auf und stellt die Wechselwirkungen von individuellen Präferenzen und Ressourcen einerseits und sozialräumlichen und situationsgebundenen Gelegenheiten andererseits in das Zentrum eines integrierten Erklärungsmodells von Jugenddelinquenz (Wikström/Treiber 2016; Wikström u.a. 2012). Dabei wird auch berücksichtigt, dass die Aktionsräume von Jugendlichen weit über das eigene Wohngebiet hinausreichen können. Das Zusammentreffen von Personen und Settings, bei dem stets ein „WahrnehmungsEntscheidungs-Prozess“ abläuft, bildet nach der SAT die unmittelbaren Ursachen von Kriminalität, während Einflüsse, die zu der Ausprägung von individuellen Verhaltenstendenzen und zur Gestaltung der Settings führen, als „causes of the causes“ eine vorgelagerte und indirekte Kausalwirkung haben. Damit werden in der SAT Elemente von Rational ChoiceTheorien und „klassischen“ sozialräumlichen sowie täterorientierten Erklärungsansätzen 
verbunden. In der Längsschnittstudie „PADS+“ (einer Stichprobe von etwa 700 Jugendlichen in Peterborough/Großbritannien) und ähnlich angelegter Schwesterstudien in den Niederlanden und Schweden wurden die theoretischen Annahmen der SAT empirisch überprüft (Wikström et al. 2010, 2012). Hinsichtlich der sozialräumlichen Dimension liegt die Besonderheit der „PADS+“-Studie in der detaillierten Erfassung der Aufenthaltsorte und der Aktivitäten aller Studienteilnehmer über mehrere Tage, so dass eine wesentlich präzisere Messung der sozialräumlichen Kontexte möglich wird, in denen Jugendliche delinquente Handlungen begehen. Die von der SAT postulierte Wechselwirkung zwischen Situationen und individuellen Neigungen konnte dadurch bestätigt werden: Delinquenz ist am wahrscheinlichsten, wenn sich Jugendliche mit delinquenten Verhaltensdispositionen an Orten aufhalten, die sowohl Gelegenheiten als auch einen Mangel an kollektiver Kontrolle aufweisen.

Methodisch stellt die empirische Untersuchung von Kontexteffekten hohe Anforderungen, denen der Großteil der älteren Forschung nicht genügen konnte (Duncan/Raudenbush 1999). Denn eine höhere Delinquenzbelastung bestimmter Sozialräume könnte bereits auf die Konzentration individueller Belastungsfaktoren (z.B. niedriger Sozialstatus, elterliches Erziehungsverhalten etc.) zurückzuführen sein. Dann würde es sich lediglich um einen Kompositionseffekt, d.h. einen Effekt der Zusammensetzung der Jugendlichen handeln. Empirische Untersuchungen zu Kontexteffekten müssen daher über sehr umfangreiche Daten sowohl zu den individuellen

als auch zu den sozialräumlichen Bedingungsfaktoren von Delinquenz verfügen und diese mit Hilfe der Mehrebenenanalyse auswerten, die eine simultane Schätzung von individuellen und kollektiven Effekten (Engel/Simonson 2005; Hummelsheim/Oberwittler/Pritsch 2015; Hox 2010). Querschnittsstudien sind ohnehin mit dem Problem belastet, dass Zusammenhänge nicht unbedingt als Ursache-Wirkungsbeziehung interpretiert werden können; Längsschnittstudien bieten überlegene Analysemöglichkeiten, sind jedoch wesentlich aufwändiger und teurer. Für die Untersuchung von Wechselwirkungen zwischen persönlichen Merkma- 
len und Kontexten oder Situationen ist zudem die Modellierung statistischer Interaktionseffekten erforderlich, auch dies ein anspruchsvolles Verfahren, bei dem häufig Fehler gemacht werden (Oberwittler/Gerstner 2015).

\section{Empirische Erkenntnisse}

\section{U.S. - amerikanische Forschung}

Bei der Darstellung der Forschungsergebnisse soll es zunächst wiederum um die vereinfachende Frage der Existenz von Verstärkungseffekten von Jugenddelinquenz durch sozialräumliche Benachteiligungen gehen, bevor anschließend der Blick auf differenzielle Effekte und Wechselwirkungen individueller und kollektiver Merkmale gelenkt wird.

Der aktuelle Wissensstand beruht überproportional auf einigen aufwändigen Längsschnittbefragungen aus den USA; die mit einem geographischen Stichprobendesign auf die Untersuchung sozialräumlicher Kontexteffekte optimiert wurden (s.u. für die Übertragbarkeit auf Europa). In einer aktuellen Metaanalyse zu sozialräumlichen Kontexteffekten auf Gewaltdelinquenz von Jugendlichen stammen 37 der 43 Studien aus den USA (Chang/Wang/Tsai 2016). Die bedeutendsten Studien sind die ,National Longitudinal Study of Adolescent Health' (,Add Health') mit ca. 20.000 Befragten und vier Befragungswellen über mehr als zehn Jahre, das ,Project of Human Development in Chicago Neighbourhoods' (PHDCN) mit ca. 6.000 Befragten und drei Befragungswellen, sowie die experimentelle Studie „Moving to Opportunity“ (MTO).

Diese Studien haben vielfache Hinweise auf die Existenz sozialräumlicher Kontexteffekte auf die Delinquenz und andere Verhaltensprobleme von Jugendlichen ergeben (Forschungsüberblicke bei Chang/Wang/Tsai 2016; Oberwittler 2013; Sharkey/Faber 2014). In der Chicagoer Längsschnittstudie erklärte die sozialräumliche Benachteiligung der Wohnquartiere $30 \%$ bis $60 \%$ der Höherbelastung der schwarzen Jugendlichen gegenüber den weißen Jugendlichen mit selbstberichteter Gewaltdelinquenz (Kirk 2008, 2009; Samp- 
son/Morenoff/Raudenbush 2005). Der in den USA bei der Erklärung von Gewalt oft im Vordergrund stehende, race'-Faktor entpuppt sich demnach zumindest teilweise als eine Folge sozialräumlicher Benachteiligungen. Auf der Basis derselben Chicagoer Daten zeigten andere Auswertungen eine signifikante Verschlechtung von

Schulleistungen, wenn sich wenige Tage zuvor in unmittelbarer Nähe ein Mord ereignet hatte (Sharkey 2010) und eine Verdoppelung der Wahrscheinlichkeit, schwere Gewalt auszuüben, wenn die Jugendlichen bis zu zwei Jahren zuvor Zeuge von Gewalt mit Schusswaffen geworden waren (Bingenheimer/Brennan/Earls 2005, vgl. Patchin et al. 2006).

Auch die ,Add Health'-Studie kann die Existenz von sozialräumlichen Verstärkungseffekten belegen. Die Armutskonzentration des Wohngebietes hat einen signifikanten Effekt auf die Gewaltdelinquenz der Jugendlichen und führt gemeinsam mit einer Reihe weiterer Risikofaktoren dazu, dass ethnische Zuordnungen statistisch gänzlich unbedeutend werden (Bellair/McNulty 2005). Auch De Coster et al. (2006: 741) kommen anhand der 'Add Health'Daten zu dem Schluss ,that the relationship between violence and individual level, race, ethnicity, poverty, parents' education, and female headship can be explained in part by the types of communities in which families and individuals reside".

Die ,Moving to Opportunity'-Studie (MTO) ist aufgrund ihrer experimentellen Anlage einmalig. Einkommensarme, meist schwarze Familien aus Wohngebieten konzentrierter Armut erhielten nach dem Zufallsprinzip Gutscheine für Umzüge in , bessere' Wohngebiete; die weitere Entwicklung der umgezogenen Familien und einer in den ursprünglichen Wohngebieten verbliebenen Vergleichsgruppe wurde über bislang fünf Jahre verfolgt. Während die Delinquenz von Mädchen in der Experimentalgruppe insgesamt abnahm, reagierten Jungen auf den Umzug zwar mit einem Rückgang der Gewaltdelikte, jedoch gleichzeitig mit einem Anstieg der Eigentumsdelikte (Burdick-Will/Ludwig 2013; Kling/Liebman/Katz 2007, Ludwig et al. 2008; siehe unten). Die Forscher erklären diesen paradoxen Befund mit der erhöhten Anreizstruktur für Eigentumsdelikte in den wohlhabenden Wohngebieten. 
Sehr bedeutsam sind auch Ergebnisse von Längsschnittstudien, die die Entwicklung von Kindern und Jugendlichen über viele Jahre weiterverfolgen und dabei sogar noch stärkere langfristige Effekte sozialräumlicher Benachteiligungen auf Delinquenz oder verwandte Verhaltensbereiche finden als Studien mit kurzem Zeithorizont, was mit kumulativen Verstärkungseffekten erklärt wird (Sharkey/Sampson 2010; .Ludwig et al. 2012; Wodtke/Harding/Elwert 2011).

\section{Europäische Forschung}

Die europäische Forschung zu sozialräumlichen Wirkungen auf Jugenddelinquenz ist viel weniger entwickelt und methodisch weniger aufwändig. Es dominierten bislang Querschnittsstudien, deren Ergebnisse zurückhaltender interpretiert werden müssen, bevor auch hier längsschnittliche Studien unter Berücksichtigung von sozialräumlichen Bedingungen begonnen wurden. Die ,MPI-Schulbefragung 1999/2000' mit ca. 5.000 befragten Jugendlichen in mehr als 60 Stadtvierteln und Schulen in Köln, Freiburg und dem Freiburger Umland war die erste deutsche Studie, die die-

sem neuen Forschungsansatz gefolgt ist (Oberwittler 2004a, 2004b, 2007a, 2007b). Als längsschnittliche Studien sind neben der bereits erwähnten englischen Studie PADS+ und ihren Schwesterstudien in den Niederlanden und Schweden auch die beiden im Ruhrgebiet angesiedelten und noch laufenden Studien „Kriminalität in der modernen Stadt“ (CRIMOC) und „Freundschaft und Gewalt im Jugendalter“ (FuGJ) zu nennen. Mehrere skandinavische Studien basieren auf der Analyse von Bevölkerungsregistern, in denen Individuen über unterschiedliche Datenbestände hinweg identifiziert und vom Kindes- bis zum Erwachsenenalter verfolgt werden können. 
Eine Reihe von Querschnittsstudien haben keine Kontexteffekte auf Jugenddelinquenz gefunden, so in Rotterdam (Rovers 1997), Antwerpen (Pauwels 2007), Peterborough (Wikström/Butterworth 2006), Duisburg (Kunadt 2010, 2013) und Hannover (Baier/Rabold 2009, Rabold/Baier 2013), während die Kölner/Freiburger Studie Hinweise auf recht starke Kontexteffekte erbrachte, die jedoch nur für einige Gruppen gelten (Oberwittler 2004a, 2004b, 2007b, siehe unten). Für einheimische Jugendliche mit auf das eigene Wohnquartier konzentriertem Freundeskreis verdoppelt sich nach dieser Studie beinahe die Wahrscheinlichkeit der schweren Delinquenz mit der Zunahme der sozialräumlichen Armut unter Kontrolle individueller Faktoren; gleichzeitig verliert der individuelle Arbeitslosen- bzw. Sozialhilfestatus der Eltern seine Signifikanz. Bei Mädchen nimmt insbesondere die Gewaltneigung stark zu, bei Jungen eher die Wahrscheinlichkeit schwerer Eigentumsdelikte. Ebenso wie in den erwähnten Analysen von Bellair/McNulty (2005) und De Coster et al. (2006) wirkt die sozialstrukturelle Benachteiligung also eher kollektiv über die Wohngebiete als individuell auf jugendliche Delinquenz. Allerdings gilt dies erwartungswidrig nicht für Jugendliche aus Migrantenfamilien, für die sich keine sozialräumlichen Verstärkungseffekte fanden (Oberwittler 2007b).

In Schweden können Forscher auf Bevölkerungsregister z.B. von Gemeinden, Schul- und Justizbehörden zurückgreifen und so methodisch einzigartige Längsschnittstudien gesamter Bevölkerungsgruppen realisieren. Mehrere registerbasierte Studien fanden nach Kontrolle der individuellen und familiären Einflussfaktoren keine sozialräumlichen Kontexteffekte (Brännström 2006; Brännström/Rojas 2012; Sarialsan et al 2013). In einer dänischen Studie jedoch errechneten Damm und Dunstmann (2014) für Flüchtlingskinder, die relativ gleichmäßig auf viele Gemeinden verteilt worden waren, eine erhöhte Wahrscheinlichkeit, später wegen eines Gewaltdelikts verurteilt zu werden, in Abhängigkeit von der Anzahl registrierter jugendlicher Straftäter, und sehen darin eine Bestätigung für Ansteckungseffekte.

Der Einfluss schulischer Kontexte auf Jugenddelinquenz wurde in den empirischen Studien seltener untersucht; teils werden Schulen unter den als wesentlicher wahrgenommen Stadtviertelkontext subsumiert. Dies macht jedoch nur insofern Sinn, als die kommunale Organisation der Sekundarschulen eine weitgehende räumliche Überlappung von Wohngebieten und Schulbezirken herstellt, was z.B. in Deutschland nicht der Fall ist (Oberwittler 2007b). 
Schulbasierte Studien konnten die Annahme bestätigen, dass die Schulorganisation und das ,Schulklima' einen eigen-

ständigen Einfluss auf das Ausmaß des delinquenten Verhaltens der Schüler (auch außerhalb der Schule) haben (Brookmeyer et al. 2006; Fuchs/Schmalz 2010; Payne 2008). Allerdings ist die Modellierung von Einflüssen des Schulklimas im Rahmen von Schülerbefragungen methodisch besonders heikel (Lütdke et al. 2009). Studien, die in sogenannten kreuzklassifizierten Mehrebenenmodellen simultan die Effekte sowohl von Wohngebieten als auch von Schulen untersuchen, deuten entweder auf stärkere (Carlson/Almquist 2016; Lecki 2009; Oberwittler 2007b) oder ausschließliche (Pauwels 2013; Pauwels et al. 2015) Effekte des Schulkontexts hin, wobei eine klare kausale Zuordnung schwierig ist (Kauppinen 2008).

Insgesamt zeichnet sich trotz der in Europa lückenhafteren Forschungslage ab, dass es wesentlich stärkere Belege für Kontexteffekte auf Jugenddelinquenz in U.S.- amerikanischen als in europäischen Studien gibt (vgl. Nieuwenhuis und Hooimeijer 2016 für Bildungserfolg). Angesichts der wesentlich stärkeren sozialen Ungleichheit, der ,Ghettoisierung' von Benachteiligungen und dem niedrigeren Niveau des Wohlfahrtsstaates in den USA ist dieses Ergebnis auch nicht überraschend, sondern spricht eher für den Erfolg des stärker wohlfahrtsstaatlich orientierten europäischen Gesellschaftsmodells.

\section{Wechselwirkungen zwischen Jugendlichen und sozialräumlichen Kontexten}

Der Forschungsschwerpunkt hat sich in den letzten Jahren zunehmend von der pauschalen Frage nach der Existenz von Kontexteffekten auf differenzierende Fragen nach den Wechselwirkungen (oder Interaktionseffekten) der individuellen Jugendlichen und ihren Familien mit den sozialräumlichen Bedingungen verlagert (Sharkey/Faber 2014; Wikström et al. 
2012). Nicht alle Jugendlichen reagieren gleich auf die sozialräumlichen Bedingungen, in denen sie leben; einige Jugendliche sind empfänglich für Gefährdungen, während andere scheinbar umempfänglich (resilient) reagieren (Elliott et al. 2006). Offenbar wirken bestimmte individuelle Eigenschaften als eine Art Puffer gegen ungünstige Umwelteinflüsse. Die Untersuchung dieser Unterschiede kann weiteren Aufschluss über die Wirkungspfade geben, die den Einfluss der strukturellen Kontextbedingungen auf das abweichende Verhalten vermitteln, und bietet Ansatzpunkte für die Gestaltung von Präventions- und Interventionskonzepten. Im Folgenden sollen einige wichtige Dimensionen, die dabei eine Rolle spielen, kurz angesprochen werden.

305

\section{Familiäre Faktoren}

Familiäre Faktoren, insbesondere die Eltern-Kind-Beziehung und das elterliche Erziehungsverhalten haben unbestritten eine zentrale Bedeutung für die Genese von Jugenddelinquenz (Farrington/Welsh 2007). Eine Reihe von Studien deuten darauf hin, dass ein positives Familienklima und Erziehungsverhalten als Schutz und ,Puffer' gegen ungünstige sozialräumliche Kontexteinflüsse wirken können. Daraus folgt umgekehrt, dass die Existenz familiärer Risikofaktoren unter ungünstigen Kontextbedingungen zu einer Verschärfung der Delinquenz führen kann. Jugendliche aus defizitären Familien in benachteiligten Wohngebieten sind demnach als besondere Risikogruppe anzusehen. Hay et al. (2007) zeigen, dass der Effekt familiärer Armut auf Jugenddelinquenz mit der Konzentration sozialer Benachteiligungen im Wohngebiet anwächst. Schonberg/Shaw (2007) kommen in einer systematischen Forschungsübersicht zu dem Ergebnis, dass in einschlägigen Studien überwiegend eine puffernde Wirkung des elterlichen Verhaltens auf die Gefährdungen des sozialräumlichen Kontextes festgestellt wurde, die allerdings in Stadtvierteln mit extremen Benachteiligungen versagt. Wenn Eltern über die Freizeitaktivitäten und -orte ihrer Kinder unterrichtet sind, hat dies besonders in benachteiligten Wohngebieten einen abschwächenden Effekt auf deren 
Delinquenz (Antunes/Lobo/Ahlin 2014; Lahey et al. 2008; vgl. auch Beyers et al. 2003; Browning/Leventha1/Brooks-Gunn 2005). Qualitative Studien haben gezeigt, dass viele Eltern große Anstrengungen unternehmen, ihre Kinder von den Gefahren der von Gewalt geprägten Armenghettos in den amerikanischen Großstädten fernzuhalten (Furstenberg et al. 1999; Molnar et al. 2005; Pettit 2004).

\section{Gleichaltrige}

Gleichaltrigenbeziehungen spielen erst seit einigen Jahren wieder eine prominente Rolle in der Forschung über Jugendkriminalität. Dazu hat unter anderem die Add Health-Studie beigetragen, die auch eine umfangreiche Netzwerkanalyse umfasst und damit vielfältige Analysemöglichkeiten bietet. Haynie/Silver/Teasdale (2006) zeigen mit diesen Daten, dass der Effekt sozialräumlicher Benachteiligungen auf Delinquenz weitgehend durch delinquente Gleichaltrigenkontakte vermittelt wird. Auswertungen von detaillierten Zeitbudget-Bögen zeigen, dass delinquentes Verhalten dann am wahrscheinlichsten ist, wenn Jugendliche sich unorganisiert mit mehreren Freunden ohne Aufsicht von Erwachsenen in öffentlichen Räumen treffen (Wikström et al. 2012). Alkohol wirkt in diesen Situationen noch als ein Verstärker (Bernasco et al. 2013). Gemeinsame unbeaufsichtigte Freizeit lässt die Wahrscheinlichkeit delinquenter Handlungen in benachteiligten Wohngebieten eher ansteigen als in anderen Wohngebieten (Bernburg/Thorlindson 2007; Maimon/Browning 2010). Die Kernannahme der SAT von der Wechselwirkung zwischen Individuum und Umwelt bestätigt sich hier: Weder verhalten sich dieselben Jugendlichen in anderen sozialräumlichen Kontexten delinquent noch andere Jugendliche in denselben sozialräumlichen Kon-

texten (Bernasco et al. 2013; Wikström u.a. 2012). 
Aber nicht alle Jugendlichen in benachteiligten Wohngebieten oder Schulen sind selbst delinquent oder haben delinquente Freunde. Vielmehr besteht bei den Gleichaltrigenbeziehungen trotz struktureller Beschränkungen stets eine Wahlmöglichkeit, die von den Jugendlichen auch genutzt wird. In der Kölner/Freiburger Studie zeigte sich, dass der räumlichen Ausrichtung der Freundeskreise und des Freizeitverhaltens eine ganz entscheidende Bedeutung für die Frage zukommt, ob Jugendliche sich von den sozialräumlichen Bedingungen ihres Wohnquartiers beeinflussen lassen oder nicht (Oberwittler 2004b). Ungefähr die Hälfte der befragten Jugendlichen haben Freundeskreise, die überwiegend außerhalb des eigenen Wohnquartiers verortet sind. Die Analysen ergaben, dass der sozialräumliche Kontext des Wohnquartiers nur für Jugendliche mit lokalem Freundeskreis bedeutsam ist; andernfalls besteht kein Zusammenhang zwischen sozialräumlicher Benachteiligung und delinquentem Verhalten. Die räumliche Auswahl der Freunde wiederum reflektiert individuelle Präferenzen der Jugendlichen gegenüber den sozialräumlichen Milieus, in denen sie leben (Arum 2000). In der Auseinandersetzung der Jugendlichen mit ihrem Wohnquartier ist demnach ein Element der Wahl und damit der Selbstselektion enthalten, das in den sozialen Brennpunkten tendenziell zu einer Spaltung in ,Anhänger' und ,Gegner' des Wohnquartiers zu führen scheint. Es sind vor allem Hauptschüler, die sich eher lokal orientieren und in den sozialen Brennpunkten an der delinquenten Subkultur teilhaben.

\section{Geschlecht}

Eines der unerwarteten, aber mittlerweile häufig bestätigten Ergebnisse der europäischen und U.S.- amerikanischen Forschung ist, dass Mädchen stärker auf sozialräumliche Kontexteinflüsse reagieren als Jungen. Dies zeigte sich nicht nur in dem bereits erwähnten ,Moving to Opportunity'-Experiment (Burdick-Will/Ludwig 2013; Kling/Liebman/Katz 2007, s.u.) und in weiteren amerikanischen Studien (Autor et al. 2015; Crowder/South 2003, Elliott et al. 2006; Fagan/Wright 2012; Haynie/Doogan/Soller 2014; Zimmerman 2016), sondern auch in der Kölner/Freiburger Studie. hier fanden sich starke sozialräumliche Kontexteffekte auf die Ge-waltneigung von einheimischen Mädchen, nicht aber von Jungen - insbesondere in Hinblick auf die Zugehörigkeit zu gewaltorientierten Cliquen -, während bei Mädchen mit Migrationshintergrund ein entgegen gerichteter Effekt zu beobachten war 
(Oberwittler 2003; 2007b). Während Mädchen aus Migrantenfamilien in den benachteiligten Stadtvierteln offenbar stärkeren familiären Kontrollen ausgesetzt sind, die ihre Gelegenheiten zu unbeaufsichtigten und riskanten Freizeitbeschäftigen begrenzen, gilt dies für einheimische Mädchen nicht. Bei ihnen scheint sich auszuwirken, dass der normative Kontext der Gleichaltrigen in den besonders benachteiligten Wohnquartieren Ge-

walthandlungen von Mädchen nicht stigmatisiert, wie es in den meisten anderen sozialräumlichen Kontexten der Fall ist. Damit übereinstimmend zeigte Kreager (2007) in einer Auswertung der schulischen Freundschaftsnetzwerke aus der „Add Health“-Studie, dass Gewalt von Mädchen in Schulen mit hohem generellen Gewaltniveau deren Populärität bei den Gleichaltrigen fördert, während gewalttätige Mädchen in allen übrigen Schulen weniger beliebt sind.

Um zu klären, warum Mädchen in der MTO-Studie deutlicher stärker als Jungen mit einer Verringerung ihrer Gewaltdelinquenz auf den Umzug von einem extrem benachteiligten Stadtviertel in ein „,besseres“ Wohnumfeld reagierten, führten die Forscher qualitative Tiefeninterviews mit 86 der beteiligten Jugendlichen in Baltimore und Chicago durch (Clampet-Sundquist et al. 2011). Es zeigte sich unter anderem, dass Jungen ihren Lebensstil weniger gut als Mädchen an die veränderten Erwartungen der Nachbarschaft anpassen konnten und ihre alten Freizeitgewohnheiten - vor allem das „Herumhängen“ mit Freunden auf der Straße - tendenziell beibehielten, was zu einer erhöhten Sichtbarkeit für die lokale Polizei und zu einer Stigmatisierung durch die Bewohner der „,bes-seren“ Wohnquartiere führte.

\section{Zusammenfassung}

Als wesentliches Ergebnis der aktuellen Forschung kann festgehalten werden, dass Kontexteffekte sozialräumlicher Benachteiligungen auf Jugendliche im Sinne einer Verstärkung der 
Delinquenz existieren, jedoch im Vergleich zu den wesentlich bedeutsameren individuellen Risikofaktoren eher schwach sind. Dies gilt erst recht im Vergleich der europäischen zu den amerikanischen Studien. Langfristige Effekte bis ins Erwachsenenalter sind vermutlich stärker als kurzfristige Effekte, was ihre Brisanz noch verstärkt. Die exakte Stärke des kausalen Effekts des Sozialraums ist aufgrund methodischer Probleme jedoch schwer zu bestimmen, da die Trennung von individuellen und kontextuellen Einflussfaktoren letztlich nicht eindeutig ist. Die Forschung hat gezeigt, dass diese Effekte des Sozialraums vorrangig über Gleichaltrigenbeziehungen vermittelt werden, und hierbei der Heterogenität von Verhaltensnormen in sozial benachteiligten Kontexten eine Schlüsselrolle zukommt.

Es ergibt sich aus den Erkenntnissen über Wechselwirkungen der sozialräumlichen Einflüsse mit individuellen Risikofaktoren, dass nur ein Teil der Jugendlichen überhaupt für Kontexteffekte empfänglich und ein nicht unwesentlicher Teil resilient ist. Dies bedeutet allerdings auch, dass die Kontexteffekte für diese ,verwundbaren' Teilgruppen von Kindern und Jugendlichen gravierender sind, als dies in den üblichen Durchschnittsberechnungen erkennbar wird. Solche differenzielle Betroffenheiten noch stärker herauszuarbeiten, sollte eine wichtige Aufgabe der zukünftigen Forschung sein.

Lassen sich aus diesen Forschungsergebnissen Folgerungen für die Prävention und Intervention von Jugenddelinquenz ziehen? Zunächst ist die Erkenntnis ent-

scheidend, dass ein Abbau von sozialen Ungleichheiten und von sozialräumlichen Konzentrationen von Benachteiligungen einen wichtigen Beitrag zur Prävention von Jugenddelinquenz leisten würde. Sozialpolitische Konzepte, die soziale Benachteiligungen abbauen und zum Erhalt des Wohlfahrtsstaates beitragen, sowie sozialräumliche Politikansätze, die die Stabilisierung oder Aufwertung benachteiligter Wohnquartiere zum Ziel haben, sind daher grundsätzlich zu begrüßen. Auch eine Reform des dreigliedrigen Schulsystems mit dem Ziel des Abbaus der sozialen Selektivität und der Konzentration benachteiligter und leistungsschwacher Jugendlicher in den Hauptschulen, die vor allem im Kontext der Schulleistungs- 
forschung gefordert wird, würde vermutlich einen wirksamen Beitrag zur Verminderung von Jugenddelinquenz leisten.

Die ,Sozialraumorientierung' spielt in der Prävention und Intervention von Jugenddelinquenz und in der Kinder- und Jugendhilfe schon lange eine bedeutsame Rolle (Deinet 2005). Die neueren Erkenntnisse über Wechselwirkungen zwischen individuellen und sozialräumlichen Risikofaktoren unterstreichen die Notwendigkeit, Maßnahmen spezifisch auf bestimmte Zielgruppen hin auszurichten, wie z. B. auf gewaltbereite Mädchen in sozialen Brennpunkten, oder migrantische Jugendliche auch außerhalb dieser Brennpunkte. Evaluationsstudien mit experimenteller Anlage, die die Wirkungen sozialpolitischer, städtebaulicher oder sozialpädagogischer Maßnahmen messen könnten, fehlen in Deutschland leider weitgehend. Angesichts der gesellschaftlichen Bedeutung einer positiven psycho-sozialen Entwicklung von Kindern und Jugendlichen sollte der Evaluation nach internationalen Standards zukünftig eine größere Bedeutung beigemessen werden. 


\section{Literatur}

Akers, R.L.; Jensen, G.F. (2003) (Hrsg.): Social learning theory and the explanation of crime: a guide for the new century. New Brunswick, N.J.

Anderson, E. (1999). Code of the street: decency, violence, and the moral life of the inner city. New York

Arum, R. (2000). Schools and communities: ecological and institutional dimensions. In: Annual Review of Sociology 26, S. 395-418

Autor, D.; Figlio, D.; Karbownik, K.; Roth, J.; Wasserman, M. (2015). Family disadvantage and the gender gap in behavioral and educational outcomes (Institute for Policy Research, Northwestern University, Working Paper 15-16).

Baerveldt, C.; Völker, B.; Van Rossem, R. (2008). Revisiting selection and influence: an inquiry into the friendship networks of high school students and their association with delinquency. In: Canadian Journal of Criminology and Criminal Justice 50(5), S. 559-587.

Baier, D.; Rabold, S. (2009). Jugendgewalt in segregierten Stadtteilen. In: Deutsche Zeitschrift für Kommunalwissenschaften(2), S. 35-49.

Baumer, E.P.; Arnio, A.N. (2012). Multi-level Modeling and Criminological Inquiry. In:

Gadd, D.; Karstedt, S.; Messner, S. (Hrsg.), The Sage Handbook of Criminological Research Methods. Los Angeles, S. 97-111.

Beier, H. (2014). Peer effects in offending behaviour across contexts: Disentangling selection, opportunity and learning processes. In: European Journal of Criminology 11(1), S. 73-90.

Bellair, P.E.; McNulty, T.L. (2005). Beyond the bell curve: community disadvantage and the explanation of black-white differences in adolscent violence. In: Criminology 43(4), S. 
$1135-1167$

Bernasco, W.; Rruiter, S.; Bruinsma, G.; Pauwels, L.; Weerman, F. (2013). Situational causes of offending: a fixed-effects analysis of space-time budget data. In: Criminology 51(4), S. 895-926.

Bernburg, J.G.; Thorlindsson, T. (2007). Community structure and adolescent delinquency in Iceland: A contextual analysis. In: Criminology 45(2), S. 415-444

Beyers, J.M.; Bates, J.E.; Pettit, G.S.; Dodge, K.A. (2003). Neighborhood structure, parenting processes, and the development of youths externalizing behaviors: a multilevel analysis. In: American Journal of Community Psychology 31(1/2), S. 35-53

Bingenheimer, J.B.; Brennan, R.T.; Earls, F.J. (2005). Firearm violence exposure and serious violent behavior. In: Science 308, S. 1323-1326

Bottoms, A.E. (2012). Developing socio-spatial criminology. In: Maguire, M.; Morgan, R.; Reiner, R. (Hrsg.), The Oxford Handbook of Criminology (5.A.). Oxford, S. 450-488

Boudon, R. (1998). Social mechanisms without black boxes. In: Hedström, P.; Swedberg, R. (Hrsg.), Social Mechanisms. An Analytical Approach to Social Theory. Cambridge, S. $172-203$

Brännström, L. (2006). Phantom of the neighbourhood. longitudinal studies on area-based conditions and individual outcomes. Stockholm

Brännström, L. (2008). Making their mark: the effects of neighbourhood and upper secondary school on educational achievement. In: European Sociological Review 24(4), S. 463478

Brännström, L.; Rojas, Y. (2012). Rethinking the Long-Term Consequences of Growing Up in a Disadvantaged Neighbourhood: Lessons from Sweden. In: Housing Studies 27(6), S. 729-747.

Brookmeyer, K.A.; Fanti, K.A.; Henrich, C.C. (2006). Schools, parents, and youth violence: a multilevel, ecological analysis. In: Journal of Clinical Child and Adolescent Psychology 35(4), S. 504-514

Browning, C.R.; Leventhal, T.; Brooks-Gunn, J. (2005). Sexual initiation in early adolescence: the nexus of parental and community control. In: American Sociological Review 
70, S. $758-778$

Burdick-Will, J.; Ludwig, J. (2013). Neighborhood Effects on Crime. Evidence from America’s Moving to Opportunity Experiment. In: Oberwittler, D.; Rabold, S.; Baier, D. (Hrsg.), Städtische Armutsquartiere - Kriminelle Lebenswelten?. Wiesbaden, S. 97-116

Bursik, R.J. (1988). Social disorganization and theories of crime and delinquency: problems and prospects. In: Criminology 26(4), S. 519-552

Carlson, P.; B. Almquist, Y. (2016). Are area-level effects just a proxy for school-level effects? Socioeconomic differences in alcohol consumption patterns among Swedish adolescents. In: Drug and Alcohol Dependence 166, S. 243-248.

Coleman, J. (1988). Social capital in the creation of human capital. In: American Journal of Sociology 94, S. 95-120

Coleman, J.S. (1990). Foundations of social theory. Cambridge, Mass.

Conger, R.D.; Ge, X.; Elder, G.H.; Lorenz, F.O.; Simons, R.L. (1994). Economic stress, coercive family process, and developmental problems of adolescents. In: Child Development 65 , S. 541-561

Crane, J. (1991). The epidemic theory of ghettos and neighborhood effects on dropping out and teenage childbearing. In: American Journal of Sociology 96, S. 1226-1259

Crowder, K.; South, S.J. (2003). Neighborhood distress and school dropout: the variable significance of community context. In: Social Science Research 32(4), S. 659-698

De Coster, S.; Heimer, K.; Wittrock, S.M. (2006). Neighborhood disadvantage, social capital, street context, and youth crime. In: Sociological Quarterly 47(4), S. 723

Deinet, U. (2005) (Hrsg.): Sozialräumliche Jugendarbeit. Grundlagen, Methoden und Praxiskonzepte. Wiesbaden

Dishion, T.J.; Tipsord, J.M. (2011). Peer Contagion in Child and Adolescent Social and 
Emotional Development. In: Annual Review of Psychology 62(1), S. 189-214.

Duncan, G.J.; Raudenbush, S.W. (1999). Assessing the effect of context in studies of child and youth development. In: Educational Psychologist 34(1), S. 29-41

Duncan, G.J.; Murnane, R. (2011) (Hrsg.): Whither Opportunity? Rising Inequality, Schools, and Children's Life Chances. New York.

Elliott, D.S.; Menard, S.; Rankin, B.H.; Wilson, W.J.; Huizinga, D. (2006). Good kids from bad neighborhoods. Successful development in social context. Cambridge

Engel, U.; Simonson, J. (2005). Sozialer Kontext in der Mehrebenenanalyse. In: Diekmann, A. (Hrsg.), Methoden der Sozialforschung. Wiesbaden, S. 303-329

Esser, H. (2002). Soziologie. Spezielle Grundlagen. 6 Bde. Frankfurt/M.

Fagan, A.A.; Wright, E.M. (2012). The effects of neighborhood context on youth violence and delinquency. does gender matter?. In: Youth Violence and Juvenile Justice 10(1), S. 64-82.

Farrington, D.P.; Welsh, B.C. (2007). Saving children from a life of crime. early risk factors and effective interventions. Oxford

Farwick, A. (2012). Segregation. In: Eckardt, F. (Hrsg.), Handbuch Stadtsoziologie. Wiesbaden, S. 381-419

Frehsee, D. (1979). Strukturbedingungen urbaner Kriminalität: Eine Kriminalgeographie der Stadt Kiel unter besonderer Berücksichtigung der Jugendkriminalität. Göttingen

Friedrichs, J. (1997). Normenpluralität und abweichendes Verhalten: Eine theoretische und empirische Analyse. In: Heitmeyer, W. (Hrsg.), Was treibt die Gesellschaft auseinander?. Frankfurt a.M., S. 473-505

Friedrichs, J.; Galster, G.; Musterd, S. (2003). Neighbourhood effects on social opportunities: The European and American research and policy context. In: Housing Studies 18(6), 


\section{S. 797-806}

Friedrichs, J.; Oberwittler, D. (2007). Soziales Kapital in Wohngebieten. In: Franzen, A.; Freitag, M. (Hrsg.), Sozialkapital. Wiesbaden, S. 450-486

Furstenberg, F.F.J.; Cook, T.D.; Eccels, J.; Elder, G.H.J.; Smeroff, A. (1999). Managing to make it. urban families and adolescent success. Chicago

Galster, G. (2012). The mechanism(s) of neighbourhood effects: theory, evidence, and policy implications. In: van Ham, M.; Manley, D.; Bailey, N.; Simpson, L.; Maclennan, D. (Hrsg.), Neighbourhood Effects Research: New Perspectives. Dordrecht; et al., S. 23-56.

Gerstner, D.; Oberwittler, D. (2015). Wer kennt wen und was geht ab? Ein netzwerkanalytischer Blick auf die Rolle delinquenter Peers im Rahmen der ,Situational Action Theory'. In: Monatsschrift für Kriminologie und Strafrechtsreform 2015(3), S. 204-226.

Grogger, J. (1998). Market wages and youth crime. In: Journal of Labour Economics 16(4), S. 756-791

Häußermann, H. (2008). Wohnen und Quartier: Ursachen sozialräumlicher Segregation. In: Huster, E.-U.; Boekh, J.; Mogge-Grotjahn, H. (Hrsg.), Handbuch Armut und Soziale Ausgrenzung. Wiesbaden, S. 335-349

Harding, D.J. (2007). Cultural context, sexual behavior, and romantic relationships in disadvantaged neighborhoods. In: American Sociological Review 72, S. 341-364

Hay, C.; Fortson, E.N.; Hollist, D.R.; Altheimer, I.; Schaible, L.M. (2007). Compounded risk: the implications for delinquency of coming from a poor family that lives in a poor community. In: Journal of Youth and Adolescence 36, S. 593-605

Haynie, D.L.; Osgood, D.W. (2005). Reconsidering peers and delinquency: How do peers matter?. In: Social Forces 84(2), S. 1109-1130

Haynie, D.L.; Silver, E.; Teasdale, B. (2006). Neighborhood characteristics, peer networks, and adolescent violence. In: Journal of Quantitative Criminology (22), S. 147-169

Haynie, D.L.; Doogan, N. J.; Soller, B. (2014). Gender, friendship networks, and delinquency: a dynamic network approach. Criminology 52(4), S. 688-722.

Haynie, D.L.; Kreager, D.A. (2013). Peer networks and crime. In: Cullen, F.T.; Wilcox, P. 
(Hrsg.), The Oxford handbook of criminological theory. New York; Oxford, S. 257-273.

Hess, H.; Scheerer, S. (2004). Theorie der Kriminalität. In: Oberwittler, D.; Karstedt, S. (Hrsg.), Soziologie der Kriminalität. Wiesbaden, S. 69-92

Hox, J. (2010). Multilevel Analysis. Techniques and Applications (2. A.). New York.

Hummelsheim, D.; Oberwittler, D.; Pritsch, J. (2015). Die Beziehung zwischen Kriminalitätsfurcht und generalisiertem Vertrauen - Mehrebenenanalysen zur Rolle individueller und kontextueller Faktoren. In: Eifler, S.; Pollich, D. (Hrsg.), Empirische Forschung über Kriminalität. Methodologische und methodische Grundlagen. Wiesbaden, S. 405-438.

Hurrelmann, K. (2006). Einführung in die Sozialisationstheorie (9. A.). Weinheim

Kauppinen, T.M. (2008). Schools as mediators of neighbourhood effects on choice between vocational and academic tracks of secondary education in helsinki. In: European Sociological Review 24, S. 379-391

Kling, J.R.; Liebman, J.B.; Katz, L.F. (2007). Experimental Analysis of Neighborhood Effects. In: Econometrica 75(1), S. 83-119

Klocke, A.; Hurrelmann, K. (1998) (Hrsg.): Kinder und Jugendliche in Armut. Opladen; Wiesbaden

Knecht, A.; Snijders, T.; Baerveldt, C.; Steglich, C.E.; Raub, W. (2010). Friendship and Delinquency: Selection and Influence Processes in Early Adolescence. In: Social Development 19(3), S. 494-514.

Kreager, D.A. (2007). When it's good to be "bad": violence and adolescent peer acceptance. In: Criminology 45(4), S. 893-923

Lahey, B.B.; Van Hulle, C.A.; Onofrio, B.M.; Lee Rodgers, J.; Waldman, I.D. (2008). Is parental knowledge of their adolescent offspring's whereabouts and peer associations spuriously associated with offspring delinquency? In: Journal of Abnormal Child Psychology 
36, S. $807-823$

Leckie, G. (2009). The complexity of school and neighbourhood effects and movements of pupils on school differences in models of educational achievement. In: Journal of the Royal Statistical Society: Series A 172(3), S. 537-554.

Ludwig, J.; Liebman, J.B.; Kling, J.R.; Duncan, G.J.; Katz, L.F.; Kessler, R.C.; Sanbonmatsu, L. (2008). What Can We Learn about Neighborhood Effects from the Moving to Opportunity Experiment?. In: American Journal of Sociology 114(1), S. 144-188.

Lüdtke, O.; Robitzsch, A.; Trautwein, U.; Kunter, M. (2009). Assessing the impact of learning environments: How to use student ratings of classroom or school characteristics in multilevel modeling. In: Contemporary Educational Psychology 34, S. 120-131.

Maimon, D.; Browning, C.R. (2010). Unstructured socializing, collective efficacy, and violent behavior among urban youth. In: Criminology 48(2), S. 443-474.Massey, D.S.; Denton, N.A. (1993). American apartheid: segregation and the making of the underclass. Cambridge/Mass.

Molnar, B.E.; Roberts, A.L.; Browne, A.; Gardener, H.; Buka, S.L. (2005). What girls need: recommendations for preventing violence among urban girls in the US. In: Social Science and Medicine 60, S. 2191-2204

Murie, A.; Musterd, S. (2004). Social exclusion and opportunity structures in european cities and neighbourhoods. In: Urban Studies 41(8), S. 1441-1459

Nieuwenhuis, J.; Hooimeijer, P. (2016). The association between neighbourhoods and educational achievement, a systematic review and meta-analysis. In: Journal of Housing and the Built Environment 31(2), S. 321-347.no

Oberwittler, D. (2003). Geschlecht, Ethnizität und sozialräumliche Benachteiligung - überraschende Interaktionen bei sozialen Bedingungsfaktoren von Gewalt und schwerer Eigentumsdelinquenz von Jugendlichen. In: Lamnek, S.; Boatca, M. (Hrsg.), Geschlecht - Gewalt - Gesellschaft. Opladen, S. 269-294 
Oberwittler, D. (2004a). A multilevel analysis of neighbourhood contextual effects on serious juvenile offending. The role of subcultural values and social disorganization. In: European Journal of Criminology 1(2), S. 201-235

Oberwittler, D. (2004b). Stadtstruktur, Freundeskreise und Delinquenz. Eine Mehrebenenanalyse zu sozialökologischen Kontexteffekten auf schwere Jugenddelinquenz. In: Oberwittler, D.; Karstedt, S. (Hrsg.), Soziologie der Kriminalität. Wiesbaden, S. 135-170

Oberwittler, D. (2007a). The effects of ethnic and social segregation on children and adolescents: recent research and results from a German multilevel study (Discussion Paper Nr SP IV 2007-603, Wissenschaftszentrum Berlin für Sozialforschung). Berlin

Oberwittler, D. (2007b). The effects of neighbourhood poverty on adolescent problem behaviours: a multi-level analysis differentiated by gender and ethnicity. In: Housing Studies 22(5), S. 781-803

Oberwittler, D. (2013). Wohnquartiere und Kriminalität - Überblick über die Forschung zu den sozialräumlichen Dimensionen urbaner Kriminalität. In: Oberwittler, D.; Rabold, S.; Baier, D. (Hrsg.), Städtische Armutsquartiere - Kriminelle Lebenswelten? Studien zu sozialräumlichen Kontexteffekten auf Jugendkriminalität und Kriminalitätswahrnehmungen. Wiesbaden, S. 45-96

Oberwittler, D.; Rabold, S.; Baier, D. (2013) (Hrsg.): Städtische Armutsquartiere - Kriminelle Lebenswelten? Studien zu sozialräumlichen Kontexteffekten auf Jugendkriminalität und Kriminalitätswahrnehmungen. Wiesbaden

Oberwittler, D.; Gerstner, D. (2015). Die Modellierung von Interaktionseffekten in Erklärungsmodellen selbstberichteter Delinquenz - Ein empirischer Vergleich von linearer OLS-Regression und negativer Binomialregression anhand der Wechselwirkungen von Risikoorientierungen und Scham. In: Eifer, S.; Pollich, D. (Hrsg.), Empirische Forschung über Kriminalität. Methodologische und methodische Grundlagen. Wiesbaden, S. 275302

Opp, K.-D. (1968). Zur Erklärung delinquenten Verhaltens von Kindern und Jugendlichen. Eine ökologische Analyse der Kinder- und Jugenddelinquenz in Köln und eine Kritik des kriminal-ökologischen Ansatzes. München

Papachristos, A.V.; Hureau, D.M.; Braga, A.A. (2013). The corner and the crew: the influ- 
ence of geography and social networks on gang violence. In: American Sociological Review 78(3), S. 417-447

Patchin, J.W.; Huebner, B.M.; McCluskey, J.D.; Varano, S.P.; Bynum, T.S. (2006). Exposure to community violence and childhood delinquency. In: Crime \& Delinquency 52(2), S. $307-332$

Pauwels, L. (2007). Buurtinvloeden en jeugddelinquentie. Een toets van de Sociale Desorganisatietheorie. Den Haag

Pauwels, L.J.; Weerman, F.M.; Bruinsma, G.J.N.; Bernasco, W. (2015). How much variance in offending, self-control and morality can be explained by neighbourhoods and schools? An exploratory cross-classified multi-level analysis. In: European Journal on Criminal Policy and Research 21(4), S. 523-537

Payne, A.A. (2008). A multilevel analysis of the relationships among communal school organization, student bonding, and delinquency. In: Journal of Research in Crime and Delinquency 45(4), S. 429-455

Payne, A.A.; Gottfredson, D.C.; Gottfredson, G.D. (2003). Schools as communities: the relationships among communal school organization, student bonding, and school disorder. In: Criminology 41(3), S. 749-777

Pettit, B. (2004). Moving and children's social connections: neighborhood context and the consequences of moving for low-income families. In: Sociological Forum 19(2), S. 285311

Rabold, S.; Baier, D. (2010). Why are some ethnic groups more violent than others? the role of friendship network's ethnic composition. In: Journal of Interpersonal Violence 26(15), S. $3127-3156$

Rovers, B. (1997). De Buurt een Broeinest? Een onderzoek naar de invloed van 
woonomgeving op jeugdcriminaliteit. Nijmegen

Sampson, R.J.; Morenoff, J.D.; Gannon-Rowley, T. (2002). Assessing "neighborhood effects": social processes and new directions in research. In: Annual Review of Sociology 28, S. $443-478$

Sampson, R.J.; Morenoff, J.D.; Raudenbush, S.W. (2005). Social anatomy of racial and ethnic disparities in violence. In: American Journal of Public Health 95(2), S. 224-232

Sampson, R.J.; Raudenbush, S.W.; Earls, F.J. (1997). Neighborhoods and violent crime: a multilevel study of collective efficacy. In: Science 277, S. 918-924

Schaefer, D.R. (2012). Youth co-offending networks: An investigation of social and spatial effects In: Social Networks 34(1), S. 141-149

Sharkey, P. (2010). The acute effect of local homicides on children's cognitive performance. In: Proceedings of the National Academy of Sciences 107(26), S. 11733-11738

Sharkey, P.; Faber, J.W. (2014). Where, when, why, and for whom do residential contexts matter? moving away from the dichotomous understanding of neighborhood effects. In: Annual Review of Sociology 40(1), S. 559-579

Schonberg, M.A.; Shaw, D.S. (2007). Do the predictors of child conduct problems vary by high- and low-levels of socioeconomic and neighborhood risk? In: Clinical Child and Family Psychology 10(2), S. 101-136

Shaw, C.; McKay, H.D. (1969 [1942]). Juvenile delinquency and urban areas. Chicago

van Ham, M.; Manley, D. (2012). Neighbourhood Effects Research at a Crossroads: Ten Challenges for Future Research (Rat für Sozial- und Wirtschaftsdaten, Working Paper Series 204)

Weerman, F.M. (2011). Delinquent peers in context: a longitudinal network analysis of selection and influence effects. In: Criminology 49(1), S. 253-286.

Wickes, R.; Hipp, J.; Sargeant, E.; Mazerolle, L. (2016). Neighborhood social ties and shared expectations for informal social control: do they influence informal social control actions? Journal of Quantitative Criminology, S. 1-29.

Wikström, P.-O.H.; Butterworth, D. (2006). Adolescent crime: individual differences and 
lifestyle risks. Cullompton

Wikström, P.-O.H. (2007). The social ecology of crime. The role of the environment in crime causation. In: Schneider, H.J. (Hrsg.), Internationales Handbuch der Kriminologie, Bd. 1: Grundlagen der Kriminologie. Berlin, S. 333-357

Wikström, P.-O.H.; Ceccato, V.; Hardie, B.; Treiber, K. (2010). Activity fields and the dynamics of crime. advancing knowledge about the role of the environment in crime causation. In: Journal of Quantitative Criminology 26(1), S. 55-86

Wikström, P.-O.H.; Oberwittler, D.; Hardie, B.; Treiber, K. (2012). Breaking rules. The social and situational dynamics of young people's urban crime. Oxford

Wikström, P.-O.H.; Treiber, K. (2016). Situational theory: The importance of interactions and action mechanisms in the explanation of crime. In: Piquero, A.R. (Hrsg.), The Handbook of Criminological Theory. Chicester, S. 415-444

Wilson, W.J. (1997). When work disappears: the world of the new urban poor. New York

Wodtke, G.T.; Harding, D.J.; Elwert, F. (2011). Neighborhood effects in temporal perspective: the impact of long-term exposure to concentrated disadvantage on high school graduation. In: American Sociological Review 76(5), S. 713-736.

Zimmerman, G.M. (2016). Examining gendered pathways in the causal chain linking neighborhood navigational strategies and unstructured socializing to adolescent violent offending. In: Journal of Criminal Justice 46, S. 94-105 